\title{
Explanation of Electromagnetic Wave and Induction by Variation of Electric Fluxline and the Deduction of Magnetism Rooted in Constancy of Light Velocity
}

\author{
Kexin Yao ${ }^{1}$ \\ ${ }^{1}$ Institute of Mechanical Engineering of Shaanxi Province, Xi'an Metering Institution, Xi'an, P. R. China \\ Correspondence: Kexin Yao, Institute of Mechanical Engineering of Shaanxi Province, Room 1-7-1, Staff \\ Building, Xi'an Metering Institution, No.12, Laodong South Road, Xi'an 710068, P. R. China. Tel: \\ 86-134-8462-6424. E-mail: yayydwpq@163.com; 029bmsp@163.com
}

$\begin{array}{lr}\text { Received: April 3, } 2014 \quad \text { Accepted: April 24, } 2014 \quad \text { Online Published: May 4, } 2014 \\ \text { doi:10.5539/apr.v6n3p112 } & \text { URL: http://dx.doi.org/10.5539/apr.v6n3p112 }\end{array}$

\begin{abstract}
According to superposition principle of electrostatic field, it is considered that electric field of free electron in electric conductor still exists and motions along with free electron. While variable motion of free electron will transmit at light velocity in electric field and form electric wave. This paper analyzed the electric field of electric wave and energy transmitted, explained and deduced law of electromagnetic induction and analyzed Maxwell equations and its physical meaning. In this paper, the author specially made deep analysis on the nature of magnetic field and concluded that constancy of light velocity is the root of magnetism.
\end{abstract}

Keywords: electric fluxline, electric wave, superposition principle, special theory of relativity, magnetic field, principle of constancy of light velocity

\section{Introduction}

The theory of electromagnetism points out that electric wave and magnetic wave exist simultaneously. Changing magnetic fields produce changing electric fields, which in turn produce changing magnetic fields. The description of such electromagnetic wave is very lofty and it is difficult for us to imagine the specific shape of such wave. Magnetism also confirms that electromagnetism wave is matter for electric wave still exists and continues transmitting after wave source stops transmitting. Then, what matter is electromagnetic wave? When magnetic induction varies, what is the intrinsic reason for induced electric field at any point in its surrounding space? Magnetism thinks that electric and magnetic fields are matters, then what relation does it have between magnetic field and electric field? And what is the nature of magnetic field? This paper uses very simple principles and deduction to answer these questions.

\section{Generation and Transmission of Electric Wave}

According to superposition principle of electrostatic field, in despite of distance between positive and negative electrons in space, the compounded electric field of these two electrons must be equal to the sum of vectors of electric field when positive and negative electrons separately exist. By inference, the separate electric field of free electrons (hereinafter known as the negatron) in any conductor and the proton (hereinafter known as positron) carrying equal electricity of free electrons still exists. Thus, when there is a changing current in the conductor, namely when negatrons in the conductor motion with variable velocity, the electric field of negatrons inevitably accompanies negatrons to motion with variable velocity. The macroscopic effect is that the negative electric field motioning with variable velocity around the conductor and static positive electric field exist at the same time. To simplify the symbols, the electric field strength generated by negatrons is represented by $\boldsymbol{E}$ and the electric field strength generated by positrons is represented by $\boldsymbol{E}_{0}$. 


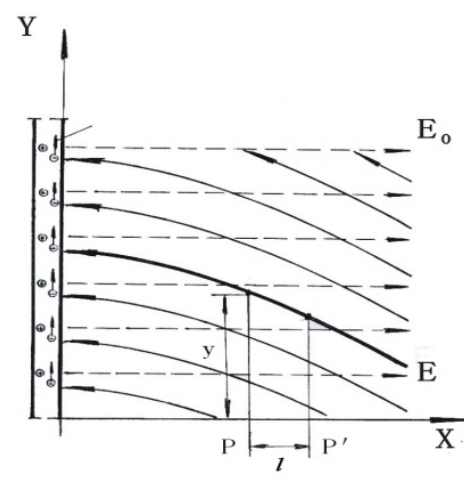

(a)

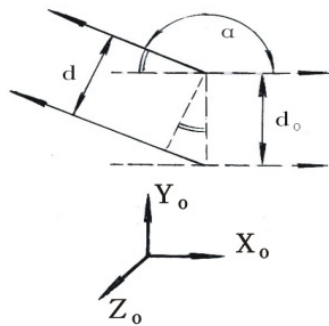

(b)

Figure 1. Distortion of electric fluxline caused by the motion of negatrons with variable velocity

Figure 1(a) represents a section of "infinitely great" plane conductor. The positrons carrying equal electric quantity as that of negatrons in conductor generate static positive electric field. The dash line represents its electric fluxline and $\boldsymbol{E}_{0}$ represents its electric field intensity. All negatrons in conductor motion with the same variable velocity and the electric field generated by negatrons also motions with them in the same variable velocity. However, as negatrons and its surrounding electric field are not the rigid combination, its motion cannot reach the infinity place instantly and will certainly transmit along $x$ direction in limited speed. Supposing this propagation velocity $d x / d t$ ( $t$ represents time) is light velocity $c$, then in case the displacement $y$ of electric field at $\mathrm{P}$ place in Figure in $y$ direction in accordance with the motion condition transmitted by negatron motion as per the law of variable velocity in Formula (1) below,

$$
y=f_{(t)}
$$

The electric field motion condition of all electric fluxlines at $\mathrm{P}$ ' point that is $l$ distance away behind $\mathrm{P}$ will be $l / c \mathrm{~s}$ behind that at $\mathrm{P}$ place. Thus, the motion law of electric field at $\mathrm{P}$ ' place shall be

$$
y=f_{(t-l / c)}
$$

In accordance with Formula (1), the motion velocity $\boldsymbol{u}$ of electric filed at P place shall be

$$
\boldsymbol{u}=\frac{d y}{d t} \boldsymbol{Y}_{o}=\frac{d f(t)}{d t} \boldsymbol{Y}_{o}
$$

Where, $\boldsymbol{Y}_{0}$ is unit vector. The $\boldsymbol{X}_{0}$ and $\boldsymbol{Z}_{0}$ in the following description are also unit vectors, please refer to Figure 1 .

As can be seen from Figure 1(a), as the variable velocity motion of negatrons transmits in limited speed $\mathrm{c}$ in electric field, the electric fluxline $\boldsymbol{E}$ of electric field of negatrons will certainly has distortion. Obviously, the distorted negatron electric field transmitted in light velocity is the generally referred electromagnetic wave.

As can be seen from Figure 1(a), the distance between $\boldsymbol{E}$ lines becomes smaller due to inclination. Figure 1(b) represents the relation between $d$ and $d_{0}$, namely, distance between inclined $\boldsymbol{E}$ line and electric field $\boldsymbol{E}_{0}$ line of positrons. In the Figure, the included angle between $\boldsymbol{E}$ and $\boldsymbol{E}_{0}$ is $\alpha$ and $d=d_{0}|\cos \alpha|$. As $\cos \alpha$ is a negative value, $d$ $=d_{0}(-\cos \alpha)$. Electric field intensity is inversely proportional to the distance between electric fluxlines, thus scalar quantity ratio of $\boldsymbol{E}$ to $\boldsymbol{E}_{0}$ is:

$$
\frac{\boldsymbol{E}}{\boldsymbol{E}_{0}}=\frac{d_{0}}{d}=\frac{1}{-\cos \alpha}
$$

That is, $\boldsymbol{E}=-\boldsymbol{E}_{0} / \cos \alpha$. We divide $\boldsymbol{E}$ into two components, one is parallel to $x$, represented with $\boldsymbol{E}_{x}$ while the other is vertical to $x$, represented with $\boldsymbol{E}_{y}$. Obviously,

$$
\boldsymbol{E}_{\mathrm{x}}=\boldsymbol{E} \cos \alpha=\frac{-\boldsymbol{E}_{0}}{\cos \alpha} \cdot \cos \alpha \boldsymbol{X}_{0}=-\boldsymbol{E}_{0} \boldsymbol{X}_{0}
$$

As $\boldsymbol{E}_{x}$ is a motional electric field, its movement velocity in $y$ direction is $\boldsymbol{u}$. Electric filed is a matter, in accordance with special relativity, the matter with movement velocity as $\boldsymbol{u}$ will contract in accordance with the proportion of $\sqrt{1-u^{2} / c^{2}}$ in movement direction. Thus, $\boldsymbol{E}_{x}$ will contract in $y$ direction in accordance with the proportion of $\sqrt{1-u^{2} / c^{2}}$. This kind of contraction will naturally make the distance between electric fluxlines 
becomes smaller. As electric field intensity is inversely proportional to the distance between electric fluxlines, the contraction of electric field $\boldsymbol{E}_{x}$ will become

$$
\boldsymbol{E}_{x}^{\prime}=\frac{\boldsymbol{E}_{x}}{\sqrt{1-u^{2} / c^{2}}}=-\frac{\boldsymbol{E}_{o}}{\sqrt{1-u^{2} / c^{2}}} \boldsymbol{X}_{o}
$$

As $\boldsymbol{E}_{y}$ has no motion in $x$ direction (Negatrons have no motion in $x$ direction in electrical conductor), thus electric filed has no contraction in $x$ direction and $\boldsymbol{E}_{y}$ has no relation with $\boldsymbol{u}$, then

$$
\boldsymbol{E}_{y}^{\prime}=\boldsymbol{E}_{y}=\boldsymbol{E} \sin \alpha=\frac{-\boldsymbol{E}_{o}}{\cos \alpha} \cdot \sin \alpha \boldsymbol{Y}_{o}=-\boldsymbol{E}_{o} \operatorname{tg} \alpha \boldsymbol{Y}_{o}
$$

In the formula above, $\operatorname{tg} \alpha$ is a negative value, thus the direction of $\boldsymbol{E}_{y}^{\prime}$ and $\boldsymbol{Y}_{\mathrm{o}}$ is the same.

$$
\boldsymbol{E}^{\prime}=\boldsymbol{E}_{x}^{\prime}+\boldsymbol{E}_{y}^{\prime}=\frac{-\boldsymbol{E}_{o}}{\sqrt{1-u^{2} / c^{2}}} \cdot \boldsymbol{X}_{o}-E_{o} \operatorname{tg} \alpha \boldsymbol{Y}_{o}
$$

The above content is the respective analysis and deduction of $\boldsymbol{E}_{x}$ and $\boldsymbol{E}_{y}$. In case $\boldsymbol{E}$ ' is directly got in accordance with transformation formula of field intensity motion as per velocity $\boldsymbol{u}$ (Yao, 2013):

The same result can be got. However, the physical significance in respective analysis is clearer. The main purpose of respective analysis is to illustrate the most basic and most important principle in this paper that electric field contracts in motion direction.

$$
\boldsymbol{E}^{\prime}=\boldsymbol{E} \sqrt{\frac{1-u^{2} \cos ^{2} \theta / c^{2}}{1-u^{2} / c^{2}}} \quad \theta \text { is the included angle between } \boldsymbol{E} \text { and } \boldsymbol{u}
$$

The above analysis indicates that electric wave actually is the process that distorted electric field makes outward transmission from wave source in light velocity and electric wave is not some kind of particular material, but the effect of combination of distorted negative electric field and positive electric field.

\section{Energy Output of Electric Wave}

It is described in above content that the separate electric fields of positrons and negatrons in conductor still exist. Electric field has electric field energy. Why there is no electric filed energy around conductor when there is no current in electrical conductor. Obviously, it is because the electric field energy of positrons and that of negatrons cancel each other out. Section 2 points out when electric wave is generated, $\boldsymbol{E}=\boldsymbol{E}_{0} / \cos \alpha,|\boldsymbol{E}|>\left|\boldsymbol{E}_{0}\right|$, the electric field energy of $\boldsymbol{E}$ is larger than that of $\boldsymbol{E}_{0}$. After cancelling out of each other, there will certainly be residual electric field energy. The residual electric field energy is the electric wave output energy. Considering the actually existing electric field energy is basically the electric field energy of negatrons, thus we define the electric field energy of negatrons as the electric field of positrons and the electric field energy of positrons as the electric field energy of negatrons. In this way, electric field energy of unit volume in electric wave shall be

$$
W=\frac{\varepsilon_{o}}{2} \boldsymbol{E}^{2}-\frac{\varepsilon_{o}}{2} \boldsymbol{E}_{o}^{2}=\frac{\varepsilon_{o}}{2}\left(\boldsymbol{E}_{x}^{2}+\boldsymbol{E}_{y}^{2}\right)-\frac{\varepsilon_{o}}{2} \boldsymbol{E}_{o}^{2}
$$

As the magnitude of electric field energy has no relation with the direction of $\boldsymbol{E}$, thus the above formula could be written as scalar expression, substitute Formula (4) into this formula and get

$$
W=\frac{\varepsilon_{0} \boldsymbol{E}_{0}^{2}}{2\left(1-u^{2} / c^{2}\right)}+\frac{\varepsilon_{o}}{2} \boldsymbol{E}_{y}^{2}-\frac{\varepsilon_{0}}{2} \boldsymbol{E}_{o}
$$

$1 /\left(1-u^{2} / c^{2}\right)$ can be expanded as $1+u^{2} / c^{2}+u^{4} / c^{4}+\ldots \ldots$ in accordance with binomial series. As $c$ is small comparing with $\mu, \mu^{4} / c^{4}$ and the various items behind it could be ignored, substitute $1 /\left(1-u^{2} / c^{2}\right)=1+u^{2} / c^{2}$ into formula above and get

$$
W=\frac{\varepsilon_{o}}{2} \boldsymbol{E}_{o}^{2}+\frac{\varepsilon_{o} u^{2} \boldsymbol{E}_{0}^{2}}{2 c^{2}}+\frac{\varepsilon_{o}}{2} \boldsymbol{E}_{y}^{2}-\frac{\varepsilon_{o}}{2} \boldsymbol{E}_{o}^{2}=\frac{\varepsilon_{0}}{2} \boldsymbol{E}_{y}^{2}+\frac{\varepsilon_{o} u^{2} \boldsymbol{E}_{o}^{2}}{2 c^{2}}=W_{1}+W_{2}
$$

In the formula, $W_{1}=\varepsilon_{o} E_{y}^{2} / 2$ represents the energy of unit volume of electric field generated by electric wave in $y$ direction. $W_{2}=\varepsilon_{o} u^{2} E_{o}^{2} / 2 c^{2}$ represents the electric field of electric wave in $x$ direction. Due to motion contraction, the energy in unit volume will increase, then what's the relation between $W_{1}$ and $W_{2}$. As $u=d y / d t, c$ $=d x / d t, \boldsymbol{E}_{y}=\boldsymbol{E}_{0} \operatorname{tg} \alpha$ and $\operatorname{tg} \alpha=d y / d x, W_{2}$ could be transformed to

$$
W_{2}=\frac{\varepsilon_{o}}{2} \boldsymbol{E}_{o}^{2} \frac{u^{2}}{c^{2}}=\frac{\varepsilon_{0}}{2} \boldsymbol{E}_{0}^{2} \cdot \frac{d y^{2}}{d t^{2}} \cdot \frac{d t^{2}}{d x^{2}}=\frac{\varepsilon_{o}}{2} \boldsymbol{E}_{o}^{2}\left(\frac{d y}{d x}\right)^{2}=\frac{\varepsilon_{o}}{2} \boldsymbol{E}_{o}^{2} \operatorname{tg}^{2} \alpha=\frac{\varepsilon_{o}}{2} \boldsymbol{E}_{y}^{2}=W_{1}
$$


Let's see the relation between $W_{2}$ and magnetic field intensity $H$.

Biot Savart Law points out the magnetic induction intensity generated by any power source Idl (the micro segment having the same direction as current $I$ in conductor) at $\boldsymbol{r}$ place is

$$
d \boldsymbol{B}=\frac{\mu_{o}}{4 \pi} \frac{I d \boldsymbol{l}}{r^{2}} \times \boldsymbol{r}_{o} \quad \text { (Unit vector in } \boldsymbol{r}_{0}-r \text { direction) }
$$

As $I$ is actually formed by the motion of free electron in conductor in velocity- $V$ (opposite to the direction of current $I$ ), representing the linear charge density in conductor with $-\tau$, then

$$
\begin{array}{r}
I d \boldsymbol{l}=-\tau(-\boldsymbol{V}) d l=d Q \boldsymbol{V} \text { as } \mu_{0} \varepsilon_{0}=1 / c^{2}, K=1 / 4 \pi \varepsilon_{0}, \text { thus } \mu_{0} / 4 \pi=K / c^{2}, \text { then } \\
d \boldsymbol{B}=\frac{K}{c^{2}} \frac{d Q \boldsymbol{V}}{r^{2}} \times \boldsymbol{r}_{o}=\frac{1}{c^{2}} \boldsymbol{V} \times d \boldsymbol{E}
\end{array}
$$

In formula above, $d \boldsymbol{E}$ represents the electric field intensity of $d Q$ at $\boldsymbol{r}$ place, this formula can also be written as

$$
\boldsymbol{B}=\frac{1}{c^{2}} \boldsymbol{V} \times \boldsymbol{E}=\mu_{o} \varepsilon_{o} \boldsymbol{V} \times \boldsymbol{E}
$$

For electric wave, the motion velocity $\boldsymbol{u}$ of $\boldsymbol{E}$ is vertical to $\boldsymbol{E}_{x}$, thus in the formula $\boldsymbol{B}=\boldsymbol{u} \times \boldsymbol{E}_{x} / c^{2}$ of magnetic induction intensity generated by $\boldsymbol{E}_{x}$, as $\boldsymbol{u}$ is vertical to $\boldsymbol{E}_{x}^{\prime}$, this formula can be expressed as scalar expression $\boldsymbol{B}$ $=u \boldsymbol{E}_{x} / c^{2}$. As in numerical value, $\boldsymbol{E}_{x}=\boldsymbol{E}_{0}$, thus $\boldsymbol{B}=u \boldsymbol{E}_{0} / c^{2}$. As $\varepsilon_{0} c^{2}=1 / \mu_{0}, H=B / \mu_{0}$,

$$
W_{2}=\frac{\varepsilon_{o} u^{2} \boldsymbol{E}_{0}^{2}}{2 C^{2}}=\frac{\varepsilon_{o}}{2} C^{2} \cdot \frac{u^{2} \boldsymbol{E}_{o}^{2}}{C^{4}}=\frac{1}{2 \mu_{o}} \boldsymbol{B}^{2}=\frac{\mu_{o}}{2} \boldsymbol{H}^{2}
$$

Substitute Formula (10) into Formula (8), and get

$$
W=W_{1}+W_{2}=\frac{\varepsilon_{o}}{2} \boldsymbol{E}_{Y}^{2}+\frac{\mu_{o}}{2} \boldsymbol{H}^{2}
$$

As $W_{1}=W_{2}$, we can get from Formula (11) that $E_{y}=H \sqrt{\mu_{0} / \varepsilon_{0}}$. In this way, Formula (11) can be transformed as

$$
W=2 W_{1}=\varepsilon_{o} \boldsymbol{E}_{y} \cdot \boldsymbol{E}_{y}=\varepsilon_{o} \boldsymbol{E}_{y} \boldsymbol{H} \sqrt{\mu_{o} / \varepsilon_{o}}=\boldsymbol{E}_{y} \boldsymbol{H} \sqrt{\mu_{0} \varepsilon_{0}}
$$

As $\sqrt{\mu_{o} \varepsilon_{o}}=1 / c$, then the above formula can be transformed as

$$
W=\frac{\boldsymbol{E}_{y} \boldsymbol{H}}{c}
$$

This $W$ represents the energy transmitted by electric wave to unit area in 1 s.

Set $\boldsymbol{S}=\boldsymbol{E}_{y} \times \boldsymbol{H}$
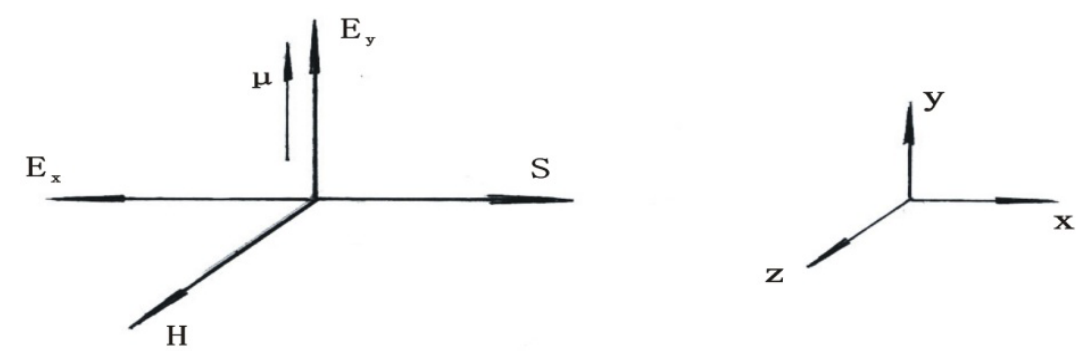

Figure 2. S. H. $\mathrm{E}_{\mathrm{y}}$ vector diagram

As $\boldsymbol{H}=\boldsymbol{B} / \mu_{0}=\boldsymbol{u} / \mu_{0} c^{2} \times \boldsymbol{E}_{x}$, its direction is vertical to $\boldsymbol{E}_{x}$ and $\boldsymbol{u}$, in $\boldsymbol{Z}$ direction, please see the Figure. $\boldsymbol{S}=\boldsymbol{E}_{y} \times \boldsymbol{H}$ is in $x$ direction, that is, the transmission direction of electric wave. Thus, $\boldsymbol{S}$ indicated the intensity of energy transmitted by electric wave to transmission direction. It is the poynting's vector defined in electromagnetism.

\section{Electric Wave and Law of Electromagnetic Induction}

Figure 3 is the simplified drawing of Figure 1. There are only two $\boldsymbol{E}$ lines in the drawing. Besides, distance $\boldsymbol{l}$ is changed to distance element $\Delta x$. Previous Formula (1) illustrates the motion law of all electric field points at $\mathrm{P}$ 
place in $y$ direction is $y_{1}=f(t)$. Previous Formula (2) illustrates the motion law of all electric field points at $P$ ' place that is $\Delta x$ distance element behind $\mathrm{P}$ in y direction shall be $y_{2}=f(t-\Delta x / c)$. Previous Formula (5) indicates the component of $\boldsymbol{E}$ in $y$ direction is $\boldsymbol{E}_{y}=-\boldsymbol{E}_{0} \operatorname{tg} \alpha \boldsymbol{Y}_{0}$.

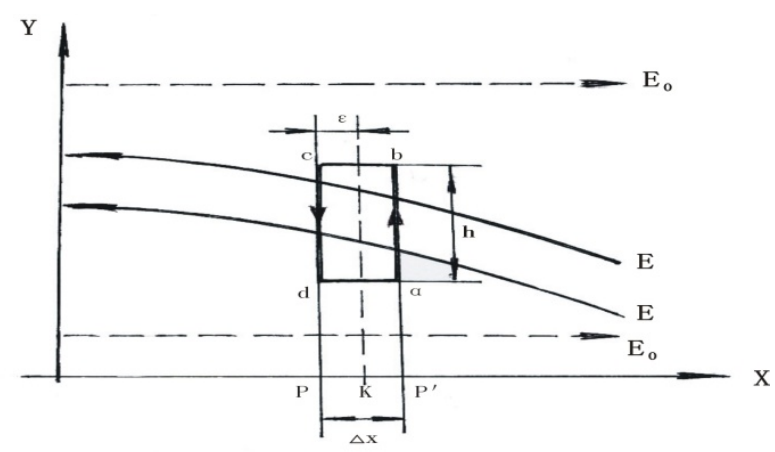

Figure 3. Electric potential generated by distortion of electric fluxline on closed loop

Item 2 points out $\cos \alpha$ is a negative value $(\alpha>\pi / 2)$, thus $\operatorname{tg} \alpha$ is also a negative value. In case $d x$ is taken as positive increment, there will be $\operatorname{tg} \alpha=-\frac{d y}{d x}=-\frac{d f(t)}{d x}$.

Substitute this formula into Formula (5) and get

$$
\boldsymbol{E}_{y_{1}}=\boldsymbol{E}_{o} \frac{d f(t)}{d x} \boldsymbol{Y}_{0}
$$

And

$$
\boldsymbol{E}_{y_{2}}=\boldsymbol{E}_{o} \frac{d f(t-\Delta x / c)}{d x} \boldsymbol{Y}_{0}
$$

Obviously, both $\boldsymbol{E}_{y 1}$ and $\boldsymbol{E}_{y 2}$ are positive values. Formulas (13) and (14) could be written as scalar expression

$$
\boldsymbol{E}_{y_{1}}=\boldsymbol{E}_{o} \frac{d f(t)}{d x} \text { and } \boldsymbol{E}_{y_{2}}=\boldsymbol{E}_{o} \frac{d f(t-\Delta x / c)}{d x}
$$

As shown in analysis diagram 3, the abcd electric potential of $l$ loop as per right-handed helical direction is

$$
\oint E \cdot d l=\int_{a}^{b} E_{y 2} d y+\int_{b}^{c}\left(E_{x}-E_{0}\right) d x+\int_{c}^{d}-E_{y 1} d y+\int_{a}^{d}\left(-E_{x}+E_{0}\right) d x
$$

Obviously, the integration of item 2 and item 4 mentioned above equals to each other and one positive and one negative cancel each other out, thus

$$
\oint E \cdot d l=\int_{a}^{b} E_{y 2} d y-\int_{c}^{d} E_{y 1} d y
$$

As all points at $\boldsymbol{E}_{y 2}$ in ab line are the same and all points at $\boldsymbol{E}_{y 1}$ in cd line are the same, and the respective length of $\mathrm{ab}$ and cd is $\mathrm{h}$, the formula above can be transformed as

$$
\oint E \cdot d l=\boldsymbol{E}_{y 2} h-\boldsymbol{E}_{y 1} h
$$

Substitute $\boldsymbol{E}_{y 1}$ and $\boldsymbol{E}_{y 2}$ in Formulas (13) and (14) into the formula above and get

$$
\begin{aligned}
\oint E \cdot d l & =\boldsymbol{E}_{o} h \frac{d}{d x} f(t-\Delta x / c)-\boldsymbol{E}_{o} h \frac{d}{d x} f(t) \\
& =-\boldsymbol{E}_{o} h \frac{d}{d x}[f(t)-f(t-\Delta x / c)]
\end{aligned}
$$

In accordance with Lagrange mean-value theorem, there is

$$
f(t)-f(t-\Delta x / c)=\left[t-\left(t-\frac{\Delta x}{c}\right)\right] \frac{d}{d t} f\left(t-\frac{\varepsilon}{c}\right)=\frac{\Delta x}{c} \frac{d}{d t} f\left(t-\frac{\varepsilon}{c}\right)
$$

In the formula above, $\varepsilon$ represents the distance element behind $\mathrm{P}, \varepsilon<\Delta x$, see Figure 3 . At $\mathrm{K}$ point, as $d f\left(t-\frac{\varepsilon}{c}\right) / d t$ expresses the y direction velocity [Formula (3)] of $\mathrm{E}$ at $\mathrm{K}$ place, make $u$ to express this velocity, 
then the above formula is transformed to

$$
f(t)-f(t-\Delta x / c)=\frac{\Delta x}{c} u
$$

Substitute the formula above into Formula (15) and get

$$
\oint E \cdot d l=-\boldsymbol{E}_{o} h \frac{d}{d x}\left(\frac{\Delta x}{c} u\right)=-\boldsymbol{E}_{o} \frac{h \Delta x}{c} \frac{d u}{d x}
$$

As $\frac{d u}{d x}=\frac{d u}{d t} \cdot \frac{d t}{d x}$

Also because $d x / d t=c$, and $h \Delta x$ is the loop abcd surrounded area, represented with $S$, then

$$
\oint E \cdot d l=-\frac{\boldsymbol{E}_{o} S}{c^{2}} \frac{d u}{d t}=-\frac{d}{d t} S \cdot \frac{\boldsymbol{E}_{o} u}{c^{2}}
$$

As in accordance with Formula (9), when $u$ is vertical to $\boldsymbol{E}_{0}, \boldsymbol{B}=\boldsymbol{E}_{0} u / c^{2}$, then the formula above is transformed to

$$
\oint E \cdot d l=-\frac{d}{d t} B \cdot S
$$

As $B \cdot S$ is abcd loop (C circuit) surrounded magnetic flux $\phi$, the formula above can be written as

$$
\oint E \cdot d l=-\frac{d}{d t} \phi
$$

Obviously, Formula (16) is the expression of law of electromagnetic induction.

\section{Analysis of Law of Electromagnetic Induction}

Figure 4(a) represents a long rectangular coil with current as $I$, we regard the left and right side of this coil as a current board respectively. The unit length of this board has $\mathrm{n}$ conductors, thus this board equals to the surface current with surface current density as $\mathrm{n} I$. In order to simplify the analysis of this question, we take the two surface plates A and B with height as $\mathrm{h}$ and between these two plates in graphical representation for analysis. A and B in Figure 4(b) represent the two current boards. The motion directions of negatrons of A and B board are opposite to the direction of $I$. As can be seen both A and B board are similar to the "infinite great" plate in Figure 1. When the current of coil has change, it equals to the variable velocity motion of negatrons in A and B board. Therefore, in accordance with above stated analysis, A and B board will transmit electric wave both to the left and right side respectively. Analyze and deduct in accordance with 4, both the electric waves transmitted by A and B board to left and right conform to Formula (16).

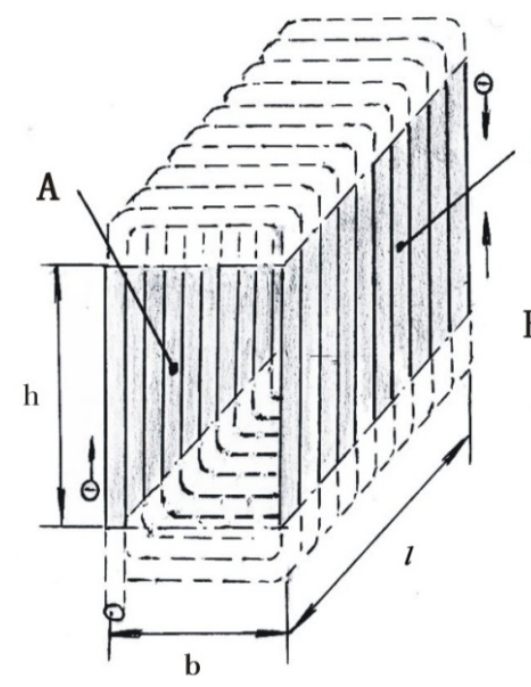

(a)

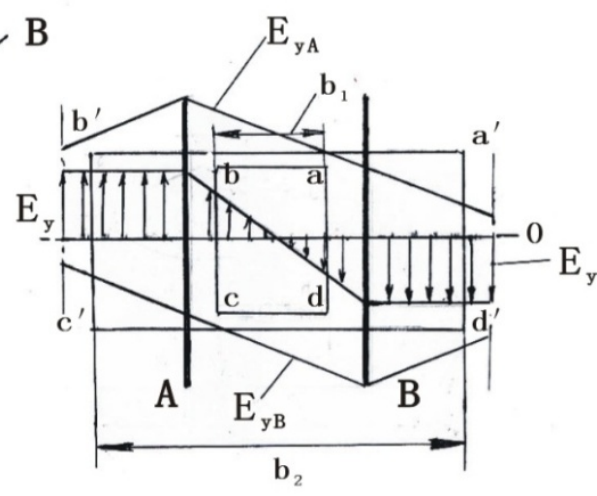

(b)

Figure 4. Electric field distribution diagrams within and outside of coil at the time of change of current 
Let us analyze the change of magnitude of $\boldsymbol{E}_{y}$ transmitted by A and B board to left and right, it is known from previous Formula (13) that

Obviously

$$
\boldsymbol{E}_{y}=\boldsymbol{E}_{o} \frac{d f(t)}{d x}=\boldsymbol{E}_{o} \frac{d y}{d x}=\boldsymbol{E}_{o} \frac{d y}{d t} \cdot \frac{d t}{d x}=\boldsymbol{E}_{o} \frac{u}{c}
$$

$$
\frac{d}{d t} \boldsymbol{E}_{y}=\frac{\boldsymbol{E}_{o}}{c} \cdot \frac{d u}{d t}=\frac{\boldsymbol{E}_{o}}{c} a
$$

$a$ in Formula (18) represents the accelerated speed when negatrons motion in variable speed, in other words, the accelerated speed of motion of negatrons decides the magnitude of change of $\boldsymbol{E}_{\boldsymbol{y}}$.

Let us analyze the electric field condition near the coil. For instance, we analyzed the condition within $3 \mathrm{~m}$ of the surrounding area. For electric wave transmitted in light velocity, the time for transmitting $3 \mathrm{~m}$ is about less than $10^{-8} \mathrm{~s}$. In such a short period of time, the accelerated speed $a$ of electric field motion near A and B board could be regarded as the same in magnitude ( $a$ is the same in $d t$ time), that is, $a$ is constant. In case $a$ is constant, $\boldsymbol{E}_{y}$ will certainly rise or reduce in accordance with $a$ proportion in $x$ direction. Set $a$ as positive, then the approaching of $\boldsymbol{E}_{y}$ far away from A board to A board rises in accordance with fixed proportion $a$. In this way, we can draw the altitude line of $\boldsymbol{E}_{y}$ magnitude on the left and right of A board, see the two straight lines at the upper part of Figure 4(b). The motion direction of negatrons of B board is opposite to that of A board, thus its $\boldsymbol{E}_{y}$ magnitude altitude line is opposite to that of A board, see the Figure. $\boldsymbol{E}_{y}$, the internal and external combination of A and B board shall be the combination of $\boldsymbol{E}_{y}$ altitude lines of A and B board. In the center of Figure 4(b), the distribution drawing of combined $\boldsymbol{E}_{y}$ is drawn. As can be easily seen from this distribution drawing that the loop abcd electric potential within A and B board (coil) is in direct proportion to width $b_{1}$, that is, the loop area is in direct proportion to the loop surrounded magnetic flux. However, the $\mathrm{a}^{\prime} \mathrm{b}^{\prime} \mathrm{c}^{\prime} \mathrm{d}^{\prime}$ loop electric potential outside of A and B board (outside of coil) has no relation with loop width $b_{2}$, that is, it has no relation with the loop area. Its electric potential equals to the electric potential when the width of loop and coil are b, namely, in direct proportion to the magnetic flux within coil.

\section{Analysis of Maxwell's Equations}

Maxwell's equations are composed of the following 4 equation sets:

$$
\begin{aligned}
& \int_{S} \boldsymbol{D} \cdot d \boldsymbol{S}=Q \\
& \oint_{1} \boldsymbol{E} \cdot d \boldsymbol{l}=-d \phi / d t \\
& \oint_{\boldsymbol{S}} \boldsymbol{H} \cdot d \boldsymbol{l}=I+d \phi_{D} / d t \\
& \int_{S}^{\boldsymbol{B}} \cdot d \boldsymbol{S}=O
\end{aligned}
$$

In Formula (a), $\boldsymbol{D}=\varepsilon \boldsymbol{E}$ is electric displacement vector. This formula is Gauss theorem. It has already been stated in physics book that Gauss theorem is derived from Coulomb's law, so we will not repeat it here. It shall be pointed out that Formula (a) is not universally true, as experiment shows there is electrostatic field in the surrounding of permanent magnet, a kind of non-charged body and the negative electrostatic field is larger than the positive electrostatic field, thus the electric displacement flux of closed surface surrounding it is not zero and Gauss theorem is false. There will be further explanation regarding this. In accordance with the inference method that there is electric field in the surrounding of permanent magnet, it can also prove that there is electrostatic field in the surrounding of current carrying coil or even current carrying conductor. Gauss theorem is proved as false again. However, for the analysis of electric wave, the electrostatic field in the surrounding of permanent magnet or current carrying coil can be ignored.

Formula (b) is law of electromagnetic induction. Section 4 and 5 above have made explanation for its source and physical significance.

In Formula (c) $\oint \boldsymbol{H} \cdot d \boldsymbol{l}=I+d \phi_{D} / d t$

It is referred to be as law of total current, in the formula, $\phi_{D}$ represents electric displacement flux. The following contents give the explanation of the source of this formula. 


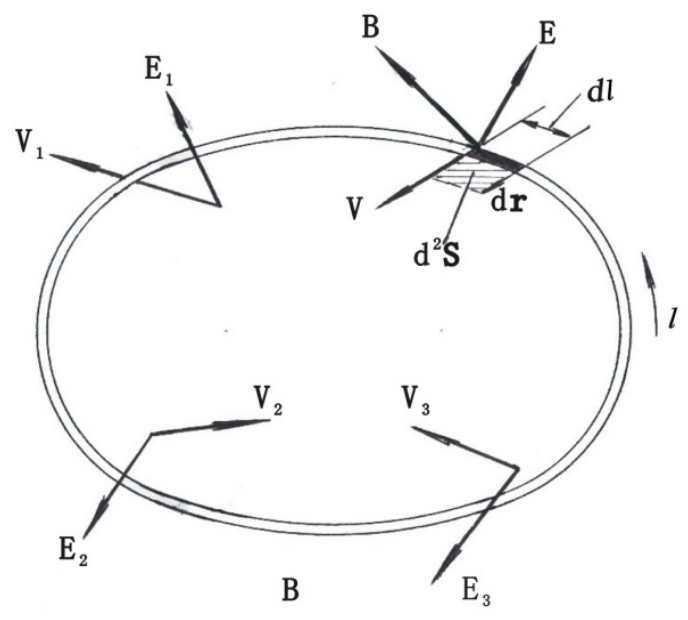

Figure 5. $d \phi_{D} / d t$ generated by motional electric field passing through $l$ loop

In Figure 5, $\boldsymbol{l}$ represents any closed loop and the winding direction is the right-handed helical direction (counter clock-wise direction). Supposing there are many electric fields with different magnitudes and motion directions in the surrounding of this circuit, $\boldsymbol{E}_{1} \boldsymbol{V}_{1}, \boldsymbol{E}_{2} \boldsymbol{V}_{2}, \boldsymbol{E}_{3} \boldsymbol{V}_{3} \ldots$. Let us analyze the motion condition of electric field in $d \boldsymbol{l}$ section (its direction is the same as that of $\boldsymbol{l}$ ) in graphical representation.

Stipulate the upward direction of electric field as positive $\boldsymbol{E}$. Positive $\boldsymbol{E}$ passes through $d \boldsymbol{l}$ from the right side of $d \boldsymbol{l}$ to the left side is positive passing through as this kind of passing through will increase the quantity of positive electric field of $\boldsymbol{l}$ loop. For the same reason, $-\boldsymbol{E}$ passes through $d \boldsymbol{l}$ from the left side of $d \boldsymbol{l}$ to the right side is also the positive passing through as this kind of passing through will reduce the quantity of negative electric field of $l$ loop. The circumstances opposite to the two mentioned above are negative passing through.

Figure 5 represents the moving $\boldsymbol{E}$ passes through $d \boldsymbol{l}$ within $d t$ in velocity $\boldsymbol{V}$ and makes ante displacement $d \boldsymbol{r}$. $d \boldsymbol{l}$ and $d \boldsymbol{r}$ form an elementary area $d \boldsymbol{l} \times d \boldsymbol{r}$ with each as the side length ( $\boldsymbol{r}$ and $\boldsymbol{V}$ are in the same direction). As both $d \boldsymbol{l}$ and $d \boldsymbol{r}$ are infinitely small quantity, the product of the two shall be second order infinitely small quantity, representing this elementary area with $d^{2} \boldsymbol{S}=d \boldsymbol{l} \times d \boldsymbol{r}$. In accordance with Formula (9), $\boldsymbol{E}$ generates $\boldsymbol{B}=\mu_{0} \varepsilon_{0} \boldsymbol{V} \times \boldsymbol{E}$ in $\boldsymbol{V}$ movement, which can also be written as $\boldsymbol{H}=\boldsymbol{B} / \mu_{0}=\varepsilon_{0} \boldsymbol{V} \times \boldsymbol{E}=\boldsymbol{V} \times \boldsymbol{D}$. The magnetic potential generated by $\boldsymbol{H}$ in $d \boldsymbol{l}$ shall be $\boldsymbol{H} \cdot d \boldsymbol{l}=(\boldsymbol{V} \times \boldsymbol{D}) \cdot d \boldsymbol{l}$ and then there shall be $(\boldsymbol{V} \times \boldsymbol{D}) \cdot d \boldsymbol{l}=(d \boldsymbol{l} \times \boldsymbol{V}) \cdot \boldsymbol{D}$ in accordance with vector formula. As $\boldsymbol{V}=d \boldsymbol{r} / \boldsymbol{d} t$, this formula can be written as

$$
\boldsymbol{H} \cdot d \boldsymbol{l}=\left(d \boldsymbol{l} \times \frac{d \boldsymbol{r}}{d t}\right) \cdot \boldsymbol{D}=\frac{1}{d t}(d \boldsymbol{l} \times d \boldsymbol{r}) \cdot \boldsymbol{D}=\frac{1}{d t} d^{2} \boldsymbol{S} \cdot \boldsymbol{D}
$$

In the formula above, $d^{2} \boldsymbol{S} \cdot \boldsymbol{D}$ is the electric displacement flux of $\boldsymbol{D}$ in $d^{2} \boldsymbol{S}$ elementary area and can be expressed with $d^{2} \phi_{D}$, thus the formula above can be transformed as $\boldsymbol{H} \cdot d \boldsymbol{l}=d^{2} \phi_{D} / d t$, then

$$
\oint \boldsymbol{H} \cdot a \boldsymbol{l}=\int_{s} d^{2} \phi_{D} / d t=d \phi_{D} / d t
$$

Obviously, $d \phi_{D} / d t$ in Formula (19) is the displacement current.

The above contents state the root cause for any part in $l$ loop generating magnetic potential $\boldsymbol{H} \cdot \boldsymbol{d l}$ is there is motional electric field at this place. This kind of analysis illustrates the internal cause of generation of electric potential and points out the physical significance of displacement current. Of course, polarization current also belongs to displacement current. As polarization current is still the electric field motion of electrons in nature, Formula (19) still true.

Let's analyze the relation between magnetic potential of loop and current in a further way. 


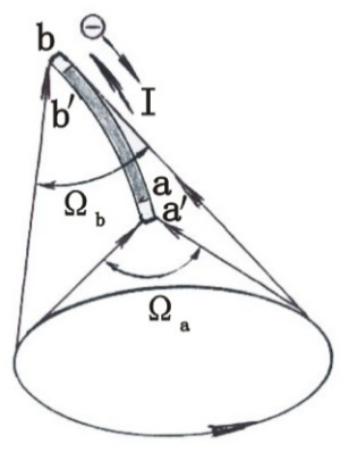

(a)

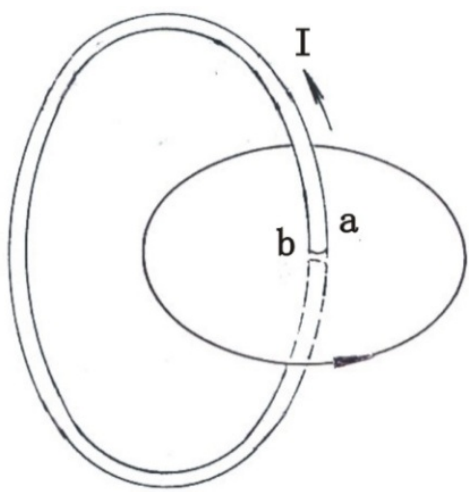

(b)

Figure 6. Demonstration of ampere circuital theorem

Figure 6(a), ab is a section of electric streamline and the direction of current $I$ is from bottom to top, but the actual motion direction of free electrons is from top to bottom. The winding direction of loop $l$ is the right-handed helical direction. Within $d t$ time, negative electron beam moves from ba to b'a'. The overlapping part of ba and b'a' negative electric beams is b'a (black and thick line in Figure). Obviously, within dt time period, the electric displacement flux generated by this section of overlapping negative electric beam b'a to $l$ loop is the same. It has no relation with the change of electric displacement flux within $l$ loop. Thus, the change caused in the electric displacement flux of $l$ loop is b'a' is bb' section smaller than ba, but aa' larger than ba. The material is conserved and the electric quantity of bb' and aa' is the same, set as $-d Q$. Obviously, within $d t$ time period, when ba negative electron beam turns into b'a' negative electron beam, the change for $l$ loop is the $-d Q$ of bb' turns to the $-d Q$ of aa'. When inferring the relation between Gauss theorem and Coulomb's law, physics has pointed out that in case electric displacement flux $d \phi_{D}$ and $d Q$ generated by $d Q$ for certain area are in direct proportion to the solid angle $\Omega$ of this area, the relational expression shall be $d \phi_{D}=\Omega d Q / 4 \pi$ (Solid angle of the whole closed surface is $4 \pi$ ). The solid angle subtended by $-d Q$ of aa' for $l$ is $\Omega_{a}$ and the solid angle subtended by $-d Q$ of bb' for $l$ is $\Omega_{b}$. The directions of electric displacement generated by $-d Q$ of aa' and bb' are upward and are positive values. Thus, within $\mathrm{dt}$ time period, the change of total electric displacement flux of $l$ loop is $d \phi_{D}=d \phi_{a a^{\prime}}-d \phi_{b b^{\prime}}=\frac{d Q}{4 \pi}\left(\Omega_{a}-\Omega_{b}\right)$.

The formula above can be transformed to $\frac{d \phi_{D}}{d t}=\frac{d Q}{d t} \frac{\left(\Omega_{a}-\Omega_{b}\right)}{4 \pi}=I\left(\frac{\Omega_{a}-\Omega_{b}}{4 \pi}\right)$.

In accordance with Formula (19), the magnetic potential of $l$ loop shall be

$$
\oint \boldsymbol{H} \cdot a \boldsymbol{l}=\frac{d \phi_{D}}{d t}=\frac{\left(\Omega_{a}-\Omega_{b}\right)}{4 \pi} I
$$

Figure 6(b) represents a closed conductor with current as I passing through $l$. We can take this closed conductor as the line from a point at the surface formed by $l$ loop to b point below this surface. In accordance with the analysis method of Formula (20), it can be regarded $\Omega_{a}=2 \pi$ and $\Omega_{b}=-2 \pi$. Thus, according to Formula (20), the magnetic potential generated by closed conductor for $l$ loop shall be

$$
\oint \boldsymbol{H} \cdot a \boldsymbol{l}=\frac{\left(\Omega_{a}-\Omega_{b}\right)}{4 \pi} I=\frac{2 \pi-(-2 \pi)}{4 \pi} I=I
$$

Obviously, Formula (21) is Ampere Circuital Theorem. It can be known by combining with Formulas (19) and (20), and considering all currents, there is

$$
\oint \boldsymbol{H} \cdot a \boldsymbol{l}=I+d \phi_{o} / d t
$$

This is the Law of Total Current, that is, Formula (c) of Maxwell's equations. It can be seen that the root of total current is the motion of electric field.

It shall be pointed out that Law of Total Current does not apply to some special circumstance. For instance, an electric charge $d Q$ passes through the center of a circular ring loop surface with radius as $r$ in velocity $\boldsymbol{V}$. At the moment of passing through the center, according to Biot-Savart Law or $d \boldsymbol{B}=\mu_{o} \varepsilon_{o} \boldsymbol{V} \times d \boldsymbol{E}$, it is obtained any point in circular ring $d \boldsymbol{H}=d Q \boldsymbol{V} / 4 \pi r^{2}$ and the magnetic potential of circular ring $\oint \boldsymbol{H} \cdot d \boldsymbol{l}=d Q V / 2 r$. There is 
no doubt that this result is correct, but this correct result cannot be inferred no matter how in accordance with Formula (c) of Law of Total Current.

In Formula (d), $\int_{S} B \cdot d s=0$

It is known that the magnetic field condition of any negatron or positron, hereinafter generally referred to be as electron, generated during motion is determined by Biot-Savart Law. It can be known from this law that the $\boldsymbol{B}$ lines produced by electrons in velocity $\boldsymbol{V}$ are all concentric circles with $\boldsymbol{V}$ as axle. Therefore, for any closed surface, in case the $\boldsymbol{B}$ line of some concentric circle penetrates out from this closed surface, this $\boldsymbol{B}$ line has to penetrate into this closed surface again, that is, the penetrating out and penetrating into equal to each other. The penetrating out $\boldsymbol{B}$ line is zero and then the total of B lines penetrating out from closed surface will certainly be zero. Thus it can be inferred that the resultant magnetic field is generated by the motion of $2,3, \ldots \mathrm{n}$ electrons, as the magnetic flux for each electron to penetrating out from closed surface is zero, the Formula (d) is true.

\section{Nature of Magnetism}

As can be seen from above analysis that magnetic field has no direct relation with the generation and transmission of electric wave and the energy transmitted by electric wave also has no direction relation with magnetic field. The reason for half of electric wave energy just to be equal to the energy of magnetic field mathematically is not the energy obtained by calculating magnetic field but the increase of field intensity caused by contraction of electric field in motion direction is calculated and electric field energy is then obtained. Besides, electromagnetic induction has never been analyzed from the change of magnetic field but the result obtained from analysis of motion and distortion of electric field. In that way, if the real material of magnetic field exist?

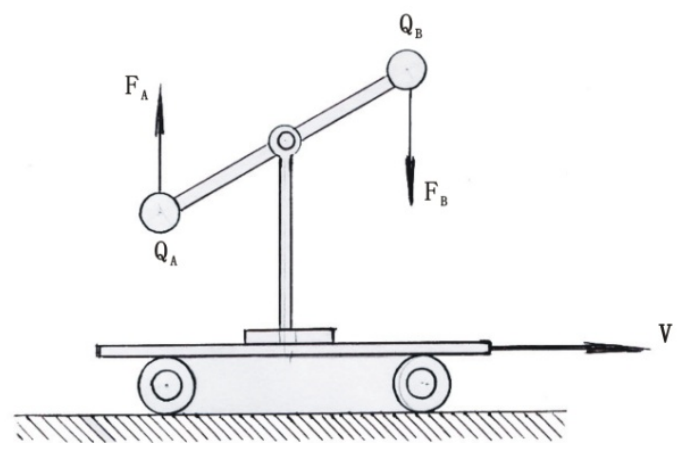

Figure 7. Observers have different opinions about whether there is magnetism or not

Figure 7 shows there is a charged body with the like electricity $Q_{A}, Q_{B}$ respectively at both ends of sway rod of stand column of moving trolley, and the sway rod is in balanced state. For people in trolley, it seems both $Q_{A}$ and $Q_{B}$ generate electrostatic field and there is only electrostatic field. However, for people on ground, there is not just electrostatic field in the surrounding of $Q_{A}$ and $Q_{B}$ on trolley, but magnetic field also exist, that is because he noticed the synergic movement of $Q_{A}$ and $Q_{B}$ and trolley, and the motion $Q_{A}$ and $Q_{B}$ will certainly generate magnetic field. This means in the same surrounding space of $Q_{A}$ and $Q_{B}$, different observer will hold opposite opinion regarding the existence of magnetic field. It shows magnetic field has essential difference with other real material, such as animal, plant, mineral, electrostatic field or gravitational field, as the existence of those real materials have no relation with observer, but the existence of magnetic field mentioned above has relation with observer.

Besides, people on ground also hold that $Q_{A}$ passes through magnetic field generated by $Q_{B}$ in velocity $\mathrm{V}$ motion and gets the upward force $F_{A}$ while $Q_{B}$ also passes through magnetic field generated by $Q_{A}$ in velocity $\mathrm{V}$ motion and gets the downward force $F_{B}$. These two forces $F_{A}$ and $F_{B}$ will make the sway rod rotate around supporting point $\mathrm{O}$ in clockwise direction. Obviously, this kind of rotation does not exist, in other words, the judgment made by people on ground by taking magnetic field as real material is wrong. This makes people have doubt about whether magnetic field is a kind of real material.

There is magnetic field in the surrounding of a permanent magnet. Theoretically, this magnetic field can be extended to infinity. In case permanent magnet motions, the magnetic field around it will certainly motion with it. In case this permanent magnet rotates in angular velocity $\omega$ around a point $O$, the surrounding magnetic field of permanent magnet will certainly rotates along with permanent magnet in $\omega$. In case magnetic field is a kind of real 
material, the rotating speed of magnetic material at some point that is $r$ distance from $\mathrm{O}$ in magnetic field will certainly be $V=\omega r$. In case $r>c / \omega$, then $V>c$. In case $r \rightarrow \infty$, then $V \rightarrow \infty$. It means the movement velocity of magnetic material can be faster than light velocity or even can be infinitely great. Some modern experiment results have proved that no material can move faster than light velocity. In case magnetic field is a real material, its motion velocity will be smaller than light velocity. However, the rotation of magnetic field means there must be the magnetic field material that is faster than light velocity. It shows magnetic field is not a real material and it can be regarded that this analysis result is a sufficient reason to deny that magnetic field is the real material (when charged body rotates, the surrounding electric field does not rotate, that's because electric field is a sourced field, when electrons rotate, its surrounding electric field only translate and does not rotate, refer to Yao (2012)).

There will certainly be some absurd deductions by taking magnetic field as the real material to analyze the question. For instance, a moving electric charge moves along the midline of coil outside of a rectangular current-carrying coil. In case it is to analyze the interaction force between coil and moving electric charge by taking magnetic field as the real material, the analysis result that acting force and anti-acting force of the two are equal in magnitude and opposite in direction will certainly be got. There is specific analysis and deduction in Yao (2012), thus we will not repeat it here. Other issue also include that observers of different inertial systems have opposite analysis and deduction for the same physical event. There is specific description in Yao (2012).

Now that the rotating question of permanent magnet cannot be solved, the error analysis of interaction force between moving electric charge and closure coil, and other unreasonable analysis cannot be changed by taking magnetic field as the real material, then how to define magnetic field if we don't take magnetic field as a material.

We analyzed and determined the Formula (9) $\boldsymbol{B}=\frac{1}{c^{2}} \boldsymbol{V} \times \boldsymbol{E}$ in accordance with Biot-Savart Law.

It can be seen from this formula that magnetic induction intensity $\boldsymbol{B}$ is the electric field intensity $\boldsymbol{E}$ motions in velocity $\boldsymbol{V}$. It is also mentioned above that in accordance with special relatively, the electric field motions in velocity $\boldsymbol{V}$ will contract in accordance with $\sqrt{1-V^{2} / C^{2}}$ proportion in motion direction. Thus, the magnetic field can be defined as magnetic field is the motional and contracting electric field and is a new material independent of electric field. In accordance with this definition of magnetic field, how shall be analyze the question by taking the magnetic field not as a material. We know that the commonly seen magnetic phenomenon is that moving electric charge will certainly get Lorentz force (It also includes the acting force applied by magnetic field on current-carrying conductor-Ampere force). Like poles of permanent magnet repel and unlike poles attract, and permanent magnet certainly attracts irons. For those really existing magnetic phenomenon, the new definition of magnetic field must be able to provide a correct answer, otherwise the new definition of magnetic field is a false one.

Figure 8(a) represents the two current boards of A and B in previous Figure 4(a). The negatron in A board moves from left to right while the negatron in B board moves from right to left (Positrons of two boards are all motionless). In Formula (9), $\boldsymbol{B}$ direction between A and B is in $\boldsymbol{Z}_{0}$ direction of graphical representation. Figure 8(a) also shows in case A and B is an integral permanent magnet (or electromagnet), the current of adjacent side of all magnetic domains (small box in Figure) within permanent magnet are in opposite direction and all cancelled out. Thus overall effect still equals to that there is only current at the upper and lower surface, which is similar to the current board of A and B.
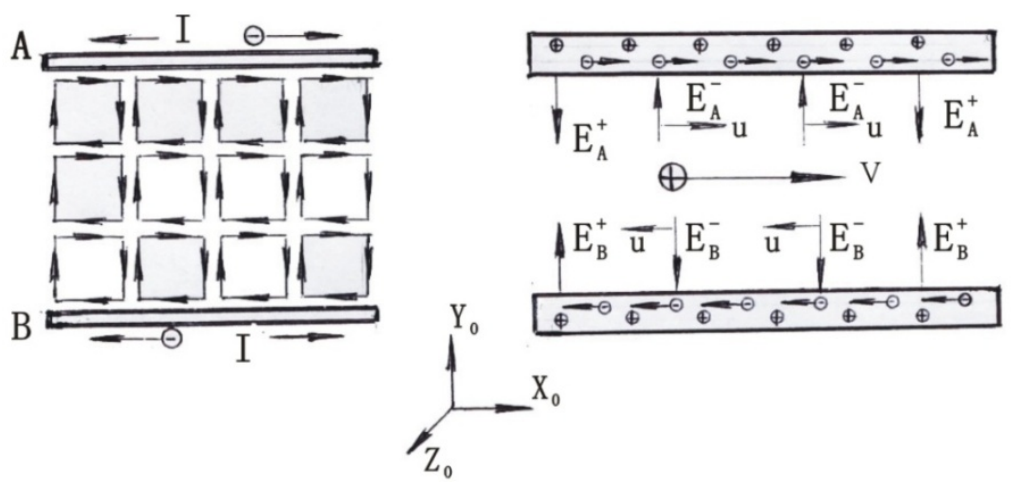

(a)

Figure 8. Analysis chart of Lorenz force 
Figure 8(b) represents the electric field condition between A and B board. The Figure shows the electric field generated by positrons of A and B board, field intensities are represented by $\boldsymbol{E}_{A}^{+}, \boldsymbol{E}_{B}^{+}$and both the two are motionless. The electric field intensities generated by negatrons of A and B board are represented by $\boldsymbol{E}_{A}^{-} 、 \boldsymbol{E}_{B}^{-}$ and both are move in the equal velocity $\boldsymbol{u}$, but in opposite direction. For A board, there is $\boldsymbol{B}_{A}=\mu_{0} \varepsilon_{0} \boldsymbol{u} \times \boldsymbol{E}_{A}^{-}$ according to Formula (9). As $\boldsymbol{u} \perp \boldsymbol{E}_{A}^{-}$and $\boldsymbol{u} \times \boldsymbol{E}_{A}^{-}$are in $\boldsymbol{Z}_{0}$ direction, thus $\boldsymbol{B}_{A}=\mu_{0} \varepsilon_{0} u E_{A}^{-} \boldsymbol{Z}_{0}$. Similarly, we can know $\boldsymbol{B}_{B}=\mu_{0} \varepsilon_{0} u E_{B}^{-} \boldsymbol{Z}_{0}$. As the absolute values of $E_{A}^{-} 、 E_{B}^{-} 、 E_{A}^{+} 、 E_{B}^{-}$are the same and equal to $\boldsymbol{E}_{0}$, it can be known total magnetic intensity induction intensity within $\mathrm{A}$ and $\mathrm{B}$ board shall be

$$
\boldsymbol{B}=\boldsymbol{B}_{A}+\boldsymbol{B}_{B}=2 \mu_{0} \varepsilon_{0} u E_{0} \boldsymbol{Z}_{0}
$$

Figure 8(b) represents a positive charge $Q$ moves along $\boldsymbol{X}_{0}$ direction between A and B board in velocity $\boldsymbol{V}$. For $Q$, it equals to $\boldsymbol{E}_{A}^{+}$of A board moves in velocity- $\boldsymbol{V}$ and $\boldsymbol{E}_{A}^{-}$moves in velocity- $(\boldsymbol{V}-\boldsymbol{u})$. Both $\boldsymbol{E}_{A}^{+}$and $\boldsymbol{E}_{A}^{-}$will contract and increase. As $1 / \sqrt{1-x^{2}}$ can be expanded as $1+x^{2} / 2+3 x^{4} / 8+5 x^{6} / 16+\ldots$ in accordance with binominal series, when $x$ is very small, $x^{4}, x^{6} \ldots$ can be ignored and there is $1 / \sqrt{1-x^{2}}=1+x^{2} / 2$. As in general condition, $\boldsymbol{u}$ and $\boldsymbol{v}$ is far smaller than c, thus the contracted and increased $\boldsymbol{E}_{A}^{+}$and $\boldsymbol{E}_{A}^{-}$can be written as

$$
\begin{gathered}
\boldsymbol{E}_{A}^{+^{\prime}}=\boldsymbol{E}_{A}^{+} / \sqrt{1-v^{2} / c^{2}}=\boldsymbol{E}_{A}^{+}\left(1+v^{2} / 2 c^{2}\right)\left(-\boldsymbol{Y}_{0}\right)=\boldsymbol{E}_{0}\left(1+v^{2} / 2 c^{2}\right)\left(-\boldsymbol{Y}_{0}\right) \\
\boldsymbol{E}_{A}^{-}=\boldsymbol{E}_{A}^{-} / \sqrt{1-(V-u)^{2} / 2 c^{2}}=E_{0}\left[1+(V-u)^{2} / 2 c^{2}\right] \boldsymbol{Y}_{0}
\end{gathered}
$$

For $Q$ of B board, $\boldsymbol{E}_{B}^{+}$and $\boldsymbol{E}_{A}^{+}$are in opposite direction and $\boldsymbol{E}_{B}^{-}$and $\boldsymbol{E}_{A}^{-}$are also in opposite direction, but the relative velocity is increased to $-[\boldsymbol{V}-(-\boldsymbol{u})]=-(\boldsymbol{V}+\boldsymbol{u})$, the contracted and increased $\boldsymbol{E}_{A}^{+}$and $\boldsymbol{E}_{A}^{-}$can be written as

$$
\begin{gathered}
\boldsymbol{E}_{B}^{+^{\prime}}=\boldsymbol{E}_{B}^{+} / \sqrt{1-v^{2} / c^{2}}=\boldsymbol{E}_{0}\left(1+v^{2} / 2 c^{2}\right) \boldsymbol{Y}_{0} \\
\boldsymbol{E}_{B}^{-^{\prime}}=\boldsymbol{E}_{B}^{-} / \sqrt{1-(\boldsymbol{V}+u)^{2} / 2 c^{2}}=\boldsymbol{E}_{0}\left[1+(\boldsymbol{V}+u)^{2} / 2 c^{2}\right]\left(-\boldsymbol{Y}_{0}\right)
\end{gathered}
$$

Total electric field intensity taken by $Q$ shall be

$$
\begin{gathered}
\boldsymbol{E}^{\prime}=\boldsymbol{E}_{A}^{+^{\prime}}+\boldsymbol{E}_{A}^{-{ }^{\prime}}+\boldsymbol{E}_{B}^{+^{\prime}}+\boldsymbol{E}_{B}^{-\prime} \\
=\boldsymbol{E}_{0}\left[\frac{(V+u)^{2}-(V-u)^{2}}{2 c^{2}}\right]\left(-\boldsymbol{Y}_{0}\right) \\
=\boldsymbol{E}_{0} \frac{2 V u}{c^{2}}\left(-\boldsymbol{Y}_{0}\right)=2 \mu_{0} \varepsilon_{0} \boldsymbol{E}_{0} V u\left(-\boldsymbol{E} \boldsymbol{Y}_{0}\right)
\end{gathered}
$$

The force acted on $Q$ is

$$
\boldsymbol{F}^{\prime}=\boldsymbol{E}^{\prime} Q=2 \mu_{0} \varepsilon_{0} E_{0} V u Q\left(-\boldsymbol{Y}_{0}\right)
$$

While Lorentz force is

$$
\boldsymbol{F}^{\prime}=Q V \times \boldsymbol{B}=Q V\left(\boldsymbol{X}_{0}\right) \times 2 \mu_{0} \varepsilon_{0} u \boldsymbol{E}_{0}\left(\boldsymbol{Z}_{0}\right)=2 \mu_{0} \varepsilon_{0} \boldsymbol{E}_{0} V u Q\left(-\boldsymbol{Y}_{0}\right)
$$

The above analysis and deduction explain the analysis result obtained by contraction of electric field in motion direction is consistent with Lorentz force.

It shall be pointed out even magnetic field is a kind of real material, Lorentz force is not applicable to inhomogeneous magnetic field either. For instance, when analyzing the interaction force between current-carrying coil and moving electric charge by utilizing Lorentz force mentioned above, there was the wrong result that the acting force and counter-acting force of the two are in the same direction but different magnitude. Take another simplest instance, in case a current element is in Y axle direction while there is an electric charge at origin of coordinates and moves along $x$ direction. Analyzing with Lorentz force, as magnetic induction intensity at origin of coordinates is zero, the force taken by moving electric charge is zero, but the magnetic induction intensity generated by moving electric charge at current element is not zero, thus the force taken by current element is not zero. Obviously, the Lorentz force analysis result that this kind of acting force does not equal to counter-acting force is wrong.

Let's analyze why like poles of permanent magnet repel and unlike poles attract. Supposing there is a bar permanent magnet respectively at the upper and lower part. The lower end of upper bar permanent magnet is pole $\mathrm{S}$ while the upper end of lower bar permanent magnet is pole $\mathrm{N}$, then the moving direction and magnitude of negatrons at these two opposite poles are the same and the two are relatively static. There is electrostatic repulsion, supposing this repulsion is defined as $-F$, then the repulsion of positrons (Carrying the same electric quantity as that 
of negatrons) of these two poles is also $-F$. Thus, total repulsion of these two opposite poles is $-2 F$.

Relative to the positrons of pole $\mathrm{N}$, the negatrons of upper pole $\mathrm{S}$ move in velocity $\mathrm{u}$ and its gravitational force shall be $\left(1+u^{2} / 2 c^{2}\right) F$ in accordance with the analysis method in previous Formula (22). In the same way, the gravitational force of negatrons of lower pole $\mathrm{N}$ for positrons of upper pole $\mathrm{S}$ shall also be $\left(1+u^{2} / 2 c^{2}\right) F$. Total gravitational force of the two shall be $2 F+u^{2} F / c^{2}$, thus total gravitational force of upper $\mathrm{S}$ and lower shall be $2 F$ $+u^{2} F / c^{2}-2 F=u^{2} F / c^{2}$.

In case the upper end of lower bar magnet is changed to pole $\mathrm{S}$, the motion direction of negatrons of the same pole $\mathrm{S}$ in opposite direction will certainly be in opposite, with relative velocity as $2 u$. The repulsion of the two shall be $-\left(1+4 u^{2} / 2 c^{2}\right) F$, thus total repulsion is increased to $-\left(1+4 u^{2} / 2 c^{2}\right) F+(-F)=-2 F-2 u^{2} F / c^{2}$. The gravitational force of negatrons of these two pole $\mathrm{S}$ for pistons is still the same and is still $2 F+u^{2} F / c^{2}$, thus the sum of repulsion and gravitational force of two pole $\mathrm{S}$ is repulsion $u^{2} F / c^{2}-2 u^{2} F / c^{2}=-u^{2} F / c^{2}$. The above analysis shows the gravitational force when unlike poles of two bar permanent magnets face with each other equals to the repulsion when like poles face with each other.

Why permanent magnet attracts irons? It is well known that there are lots of magnetic domains within iron material. The unlike pole of magnetic domain is attracted by the like pole of permanent magnet while the like pole of magnetic domain repulses by the same pole of permanent magnet. This kind of attraction and repulsion make the magnetic domain moves and cause the unlike pole is close to permanent magnet while the like pole is far away from permanent magnet. As attraction is larger than repulsion, thus irons are attracted.

Since magnetic field is not a material, why magnetic field has energy? This question has been pointed out when analyzing energy of electric wave. In effect, the so-called energy of magnetic field is the energy increased by the contraction and motion of electric field. Please refer to the source deduction in previous Formula (10).

Let's analyze the interaction force between moving electric charge and current-carrying coil. It has been pointed out in previous content that the result that acting force does not equal to counter-acting force will certainly be got by analyzing the interaction force between moving electric charge and current element with Lorentz force. Obviously, this error in basic analysis is the root cause for the error of the whole. Whether there is the error that acting force does not equal to counter-acting force when analyzing the mutual acting force of two moving electric charges by applying the principle that electric field makes length contraction in motion direction.

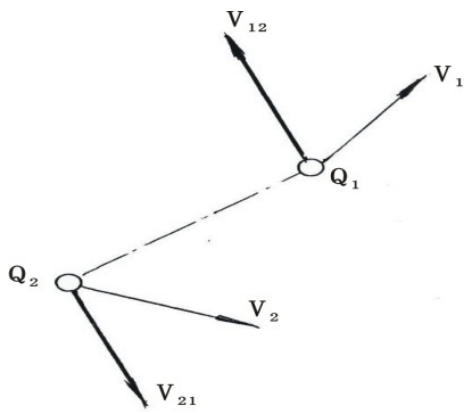

(a)

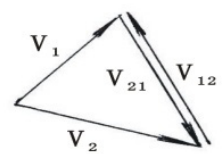

(b)

Figure 9. Interaction force between two moving electric charges

Figure 9(a) shows two electric charges $Q_{1}$ and $Q_{2}$ move in velocity $V_{1}$ and $V_{2}$ respectively. Figure 9 (b) represents velocity composition diagram of $V_{1}$ and $V_{2}$. Obviously, the relative movement velocity $V_{12}$ of $Q_{1}$ to $Q_{2}$ and the movement velocity $Q_{2}$ of $Q_{2}$ are equal in magnitude but different in direction, thus the contraction condition of electric field of $Q_{1}$ relative to $Q_{2}$ is the same as the contraction condition of electric field of $Q_{2}$ relative to $Q_{1}$, and electric fields of the two change in accordance with the same proportion. It is because the acting forces of the two equals to counter-acting forces, after changing as per the same proportion, the acting forces still equal to counter-acting forces. The connecting line between $Q_{1}$ and $Q_{2}$ also represents the electric field intensity line between the two. It is quite clear that the electric field force taken by $Q_{1}$ from $Q_{2}$ electric field and the electric field force taken by $Q_{2}$ from $Q_{1}$ electric field are in opposite direction. It can be known through analysis by summing up the two points stated above that when analyzing the mutual acting forces between any two electric charges with the principle that electric field makes length contraction in motion direction, the result that acting forces and the counter-acting forces of the two are constantly equal in magnitude but opposite in direction. 
Therefore, the interaction forces between all positrons and all negatrons of moving electric charges and current-carrying coil will certainly be equal in magnitude but opposite in direction. The overall analysis result will certainly be the mutual acting forces between moving electric charges and current-carrying coil are equal in magnitude but opposite in direction.

Does the new principle that magnetic field is the motional and contracting electric field has any new deduction? It can be seen from previous discussion that here are some new deductions. The most obvious one is in accordance with the principle that electric field makes length contraction in motion direction, the moving negatrons in a current-carrying conductor will certainly make length contraction in motion direction, which then make the negative electric field of negatrons in conductor is larger than the positive electric field of positrons. The macro-effect is there is negative electric field in the surrounding of conductor. However, this deduction cannot be verified now, that is because the movement velocity of negatrons (free electrons) in conductor is very low and generally lower than $10^{-2} \mathrm{~m} / \mathrm{s}$, thus its electric field contraction is very small, which is hard to be detected. However, the spinning velocity of electrons in magnetic domain of permanent magnet, especially of $\mathrm{NdFeB}$ permanent magnet could reach $10^{6} \mathrm{~m} / \mathrm{s}$. Thus in $\mathrm{NdFeB}$, the contraction of electric field caused by the electron motion within magnetic domain is very large and there shall be the existence of macro electric field outside of permanent magnet. In 2004, I measured the condition of electric field around cubic NdFeB permanent magnet with the side length as $0.02 \mathrm{~m}$. Under the condition that the magnetic induction intensity at the surface of permanent magnet is about $0.8 \mathrm{~T}$, it is measured the intensity of negative electric field at $0.01 \mathrm{~m}$ of the upper part of four edges of pole $\mathrm{N}$ and B surface is about $-243 \mathrm{v} / \mathrm{m}$; at the $0.02 \mathrm{~m}$ of upper part, the intensity of negative electric field is about $-133 \mathrm{v} / \mathrm{m}$, while at $0.01 \mathrm{~m}$ of the upper part of four edges of the four side faces of permanent magnet, it is measured the positive electric field intensity is $196 \mathrm{v} / \mathrm{m}$ and at $0.02 \mathrm{~m}$ of upper part, the intensity of positive electric field is about $97 \mathrm{v} / \mathrm{m}$. however, electric field can neither be detected at the place above center of pole $\mathrm{N}$ and $\mathrm{S}$ nor at the place above center of side face. By applying the opinion that electric field makes length contraction in motion direction, I made the analysis and research of the possibility of electric field existence around permanent magnet for a long time and Mr. Wang Zhisheng helped me did a lot of deduction work. The analysis result shows the analysis conclusion obtained by utilizing the opinion that electric field makes length contraction in motion direction is basically consistent with experiment result. Please refer to Yao and Wang (2012) for specific analysis and deduction condition.

It can be regarded the experimental result that electric field exists in the surrounding of permanent magnet proved the opinion that electric field makes length contraction in motion direction is also true and also proved that magnetism is not a new material independent of electric field. It is just the superficial phenomenon of moving and contracting of electric field.

\section{Deduction}

It can be obviously seen from the analysis of Lorentz force in Section 7, the analysis that like poles of permanent magnet repel while unlike poles attract, and the deduction process in Section 3 for magnetic field energy that the basic basis of those analysis and deduction processes is the important principle that electric field makes length contraction in motion direction, while magnetic induction intensity $\boldsymbol{B}=\varepsilon_{0} \mu_{0} \boldsymbol{V} \times \boldsymbol{E}$, the formula reflecting magnetic field condition cannot play the obvious function for analyzing and deducting the function of magnetism. This shows that if the objective law that electric field makes length contraction in motion direction does not exist, neither Lorentz force nor energy of magnetic field exists... In other words, magnetism will not exist and then there will be no modern technology such as TVs, computers or remote controllers without magnetism. Why electric field makes length contraction in motion direction? Naturally, it is the theoretical deduction of special relativity while the basis for establishment of special relativity is Lorentz transformation and the experimental basis of Lorentz transformation is the mysterious law (principle) of constancy of light velocity. It shows without constant light velocity, there will be no magnetism and then no modern technology. Thus, our important deduction is the law (principle) of constancy of light velocity is the key given by God to human beings for opening the gate to modernization.

Let's analyze the transmission velocity of electric wave-light velocity $c$. People naturally hope the faster the light velocity is, the better. As when light velocity becomes faster, signal transmission and receiving will be faster and astronomical observation will be more convenient and favorable. However, it is not so good when light velocity is too fast. As previous Formula (17) shows the intensity of signal transmitted by electric wave $\boldsymbol{E}_{y}$ $=\boldsymbol{E}_{0} \mu / c$, under the condition that movement velocity of negatrons of wave source is constant, the larger the $c$ is, the smaller the $\boldsymbol{E}_{y}$ is. Formula $\mu_{0}=1 / \varepsilon_{0} c^{2}$ shows the intensity of magnetic phenomenon is inversely proportional to $c^{2}$. In case $c$ is infinitely great, there will be no magnetism and then no electric wave. For instance, when light velocity increases from $3 \times 10^{8} \mathrm{~m}$ to $3 \times 10^{9} \mathrm{~m}, c^{2}$ will be 100 times larger while $\mu_{0}$ will be 100 times smaller. Such 
a small $\mu_{0}$ will make the signal of electric wave very weak and people might not have invented wireless communication, thus we don't hope light velocity to become faster any more. However, it is not good either when light velocity is too smaller. When light velocity becomes small, not only the transmission and receiving of signal will become slow, but $\boldsymbol{E}_{y}$ will increase and $\mu_{0}$ will accelerate and rise, and the electromagnetic radiation and interference will be greatly enhanced, which is unfavorable for human beings. When it becomes serious, it can be even unfavorable for the survival of human beings or other living beings. Thus we don't hope light velocity to become smaller any more. Obviously, the light velocity felt by us in reality is the most favorable light velocity. In this way, the second important deduction we got is the $300,000 \mathrm{kilo} / \mathrm{s}$ light velocity is the most ideal light velocity given by God to our human beings.

\section{References}

Guo, S. H. (2010). Electrcdynamics. China: Higher Education Press.

Yao, K. X. (2012). Question on some principles of electromagnetism. Applied Physics Research, 4(3), 115-122. http://dx.doi.org/10.5539/apr.v4n3p115

Yao, K. X. (2013). Set up invariable axiom of force equilibrium and solve problems about transformation of force and gravitational mass. Applied Physics Research, 5(1), 72-77. http://dx.doi.org/10.5539/apr.v5n1p72

Yao, K. X., \& Wang, Z. S. (2012). Inferring the fact that static magnetic field exists along with electrostatic field and conducting experimental verification in accordance with the theory of relativity. Applied Physics Research, 4(1), 222-240. http://dx.doi.org/10.5539/apr.v4n1p222

Zhang, Z. H. (2005). Fundamentals of Principle of Relativity. Beijing: Tsinghua University Press.

\section{Copyrights}

Copyright for this article is retained by the author(s), with first publication rights granted to the journal.

This is an open-access article distributed under the terms and conditions of the Creative Commons Attribution license (http://creativecommons.org/licenses/by/3.0/). 